\title{
Telemedicine Forum being established
}

$\mathrm{W}_{\mathrm{e}}$ e are told that information is the currency of the Nineties. Information couldsimply be regarded as any data which is useful.A significant advantage of information in digital format is the ability to utilise that information at two or more differentlocations, markedly increasing its usefulness. Intrinsic to the information revolution over the preceding decadeshavebeen advancesin computer and telecommunicationstechnology. The single overriding factor in the success of theseinformation technology industries was the use of standardised hardware and software components, ensuring compatibility between a variety of manufacturers, permitting theindustry's phenomenal growth. These standards include mechanical and electrical connections, transmission media, electronic signal characteristics and sophisticated software protocols and applications.

The ability to capture, process and store information in digital formathas aready had a huge impact on Radiology.Just as in information technology the development of medical and radiological standards at various strata has been a critical element in allowing the cohesive and productive evolution of technical advancesin our profession.

Thesameneed for standardsmustapply to the rapidly germinating field of telemedicine, as a specialized hybrid branch of information technology and medicine. Again multiple layers of standards and protocols are required covering physical, electrical and software aspects, butbeyond that are the medical and medicolegal considerations.
Thereareatleast threegoodpractical reasons for developing locally accepted standards - firstly, to protect the public and the medical profession; secondly, to allowintercommunication between systems; and thirdly, to avoid obsolescence.Already examples of inferior performance of teleradiology systems have raised thelevel of anxiety concerning medicolegal action.Standards may well differ according to individual circumstances of specific telemedicine systems, with the various permutations of potential links between and within hospitals, practices, clinics and homes.

Should a set of basic SouthAfrican hardware and software standards addressing medical image transmission be published? Should these be verified and certification be implemented? One would envisage that DICOM3 standardswould be non-negotiable.Digital image andmonitor specifications would probably bethenextmostimportant, requiring at leasta 2000 by 2000 matrixsize and 12 bits of contrast resolution for diagnostic chest radiology (and possibly a 4000 by 4000 matrix size for mammography), ideally with high luminance, flicker-freescreens.

Compressionisamorecontentiousissue, particularly whether it is permissible to allow "lossy" compression with the attendant irreversibleloss ofdata.Intercommunication between varioussystems and future upgrade potential wouldbesharedconcems,probably ultimately determined by marketforces.

Following a meeting of interested parties in CapeTown on 20January 1996, proposals and discussion on these topicsled to the formation of a steering committee on telemedicine which has as its objective the establishment of a National Forum on Telemedicine.

This committee presently consists of $\mathrm{Dr}$ Jan Lotz (chairman), ProfSteve Beningfield (UCT), Dr PieterSerfontein (Provincial administration Western Cape), Dr Ashwin Hurribunce (SANDF), Dr Stuart Rutherfoord (Pathologist, University Stellenbosch) andDrLennoxMathews(Advisor,Dept of National Health). Private practice, state, provincial, military and academic groups are therefore represented and both radiology and pathology specialties are included. Any representatives from other interested groups or specialities would be warmly welcomed. Theintention of the proposed forum is to represent all interested parties, acting as a conduit between users of telemedicine and central government. The Department of Health has already constituted a committee evaluating the appropriateness and application of telemedicine in this country and has been informed of the planneddevelopment of theforum. Thisgovernmental committee would be the most likely avenue of communication with central structures, and many of these issues are perhapsmoreappropriately addressed at that level. We hope that the proposed national telemedicine forum will serve to help cultivate the discipline required to prevent the devaluation of our own unique currency, medicalinformation.

A South African National
TelemedicineForum homepage can
be accessed on Internet at the follow-
ing address: http://www.sun.ac.za/
local/academic/Anat_Path/
neuro_path/telemed.html

JLotz

SJBeningfield 